\title{
Longitudinal Optical Phonons Modified by Organic Molecular Cation Motions in Organic-Inorganic Hybrid Perovskites
}

\author{
Masaya Nagai,${ }^{1, *}$ Takuya Tomioka, ${ }^{1}$ Masaaki Ashida, ${ }^{1}$ Mizuki Hoyano, ${ }^{2}$ Ryo Akashi, ${ }^{2}$ \\ Yasuhiro Yamada, ${ }^{2, \dagger}$ Tomoko Aharen, ${ }^{3}$ and Yoshihiko Kanemitsu ${ }^{3, \$}$ \\ ${ }^{1}$ Graduate School of Engineering Science, Osaka University, Toyonaka 560-8531, Japan \\ ${ }^{2}$ Department of Physics, Chiba University, Inage-ku, Chiba 263-8522, Japan \\ ${ }^{3}$ Institute for Chemical Research, Kyoto University, Uji, Kyoto 611-0011, Japan
}

(Received 15 January 2018; revised manuscript received 3 August 2018; published 5 October 2018)

\begin{abstract}
We performed terahertz time-domain spectroscopy for methylammonium (MA) lead halide perovskite single crystals and characterized the longitudinal optical (LO) phonons directly. We found that the effective LO phonon wave number does not change in the wide temperature range between 10 and $300 \mathrm{~K}$. However, the coupling between MA cation modes and the LO phonon mode derived from lead halide cages induces a mode splitting at low temperatures and a damping of the LO phonon mode at high temperatures. These results influence the interpretation of electron-LO phonon interactions in perovskite semiconductors, as well as the interpretations of mobility, carrier diffusion, and polaron formation.
\end{abstract}

DOI: 10.1103/PhysRevLett.121.145506

The organic-inorganic hybrid lead halide perovskites with the $\mathrm{APb}_{3}(A=$ monovalent cation and $X=\mathrm{I}, \mathrm{Br}, \mathrm{Cl})$ structure are a class of promising materials for solutionprocessed high-efficiency solar cells [1]. One of the most typical compositions is the $\mathrm{CH}_{3} \mathrm{NH}_{3}$ (methylammonium, MA) lead halide perovskite $\mathrm{MAPb}_{3}$, which exhibits various attractive properties such as a sharp direct-gap absorption edge and long carrier diffusion [2,3]. Furthermore, these properties also allow implementation in other important optoelectronic devices such as lightemitting diodes [4], lasers [5], and phototransistors [6].

The deep understanding of the charge-carrier interactions in $\mathrm{MAPb} X_{3}$ is essential to exploit their fascinating optoelectronic properties in novel optoelectronic devices. In particular, the long-wavelength longitudinal optical (LO) phonon involves a uniform internal electric field due to the macroscopic motion of charged atoms, which enhances Coulomb interaction with the carriers [7]. This is the socalled Fröhlich interaction, and governs the carrier's mobility [8], its energy dissipation process [9], the phonon bottleneck that slows down the hot-carrier cooling [10], and the spectral bandwidth of the luminescence at room temperature [11,12]. LO phonons in $\mathrm{MAPb} X_{3}$ have been characterized by Raman spectroscopy [13], terahertz (THz) spectroscopy [14-17], and photoluminescence (PL) measurements $[11,12]$. However, the reported values of the

Published by the American Physical Society under the terms of the Creative Commons Attribution 4.0 International license. Further distribution of this work must maintain attribution to the author(s) and the published article's title, journal citation, and DOI.
LO phonon are controversial since they are ranging from 91 to $133 \mathrm{~cm}^{-1}$ for $\mathrm{MAPbI}_{3}$ and from 118 to $167 \mathrm{~cm}^{-1}$ for $\mathrm{MAPbBr}_{3}$. This large variation obscures the essential information for the Fröhlich interaction and thus makes correct interpretation of the physics more difficult in these materials.

Recent studies revealed that the dynamical motion of the $A$-site MA cations also strongly influences the optoelectronic properties of the perovskites [18]. A direct influence on the LO phonon would explain several interesting features, but experimental evidence is lacking. Single crystals seem to be more suited for this task because the polycrystalline grains in thin films may induce a morphology dependence [19-22]. Furthermore, the high-temperature tetragonal phase remains in some thin films even below the phase transition temperature, and this affects the accuracy of the analysis [23-27]. The lead halide cages are deformed in the low-temperature orthorhombic phase [28], which strongly restricts the rotational motion of the MA cations. However, the lattice expansion in the tetragonal and cubic high-temperature phases allows the free rotation of the MA cations between the lead halide cages. This implies structural fluctuations and dynamical screening, which may influence the carrier mobility, tolerance of carrier trapping, and the stability of excitons [3,11,12,29,30]. Fast rotation of the MA cation has been revealed with NMR spectroscopy [31], twodimensional spectroscopy [32], and quasielastic neutron scattering measurements [33], and the reported timescale of picoseconds is comparable to the period of the LO phonon.

In this Letter, we performed $\mathrm{THz}$ time-domain reflection spectroscopy for $\mathrm{MAPb} X_{3}$ single crystals $(X=\mathrm{I}, \mathrm{Br}, \mathrm{Cl})$ 
below $230 \mathrm{~cm}^{-1}$ at various temperatures. The reflection measurements allowed us to evaluate the dielectric constants $\epsilon$ of the strongly absorbing samples with high dynamic range [34]. From the energy loss function $\operatorname{Im}(-1 / \epsilon)$, which directly represents the longitudinal excitation [35], we found for the first time that the MA cations' modes modify the LO phonon modes at low temperatures. The comparison of $\mathrm{THz}$ data and temperature-dependent PL spectra reveals that there is no significant influence of the LO phonon, which is altered by the MA cations, on the electron-phonon scattering processes. It is known that the selection of a proper $A$-site cation is critical in device design because it determines the chemical and physical stability [36]. Our results indicate a significant contribution of the $A$-site MA cation motion to the Fröhlich interaction, and will propose important details for the Fröhlich interaction in organic-inorganic hybrid perovskite semiconductors.

Figure 1(a) shows the reflection spectra $R$ for the $\mathrm{MAPbI}_{3}$ single crystal at room temperature (red curve) and low temperature (blue curve). The sample preparation and setup of the THz reflection spectroscopy are described in Ref. [37]. At $300 \mathrm{~K}$, the sample has a tetragonal crystal structure and two reflection peaks appear at 33 and $71 \mathrm{~cm}^{-1}$. These peaks are attributed to the Reststrahlen bands for polar materials $[16,44]$. At $10 \mathrm{~K}, \mathrm{MAPbI}_{3}$ is in the orthorhombic phase and additional reflection peaks appear at 82, 95, 103, and $161 \mathrm{~cm}^{-1}$. We also show the reflection spectra of the $\mathrm{MAPbBr}_{3}$ and $\mathrm{MAPbCl}_{3}$

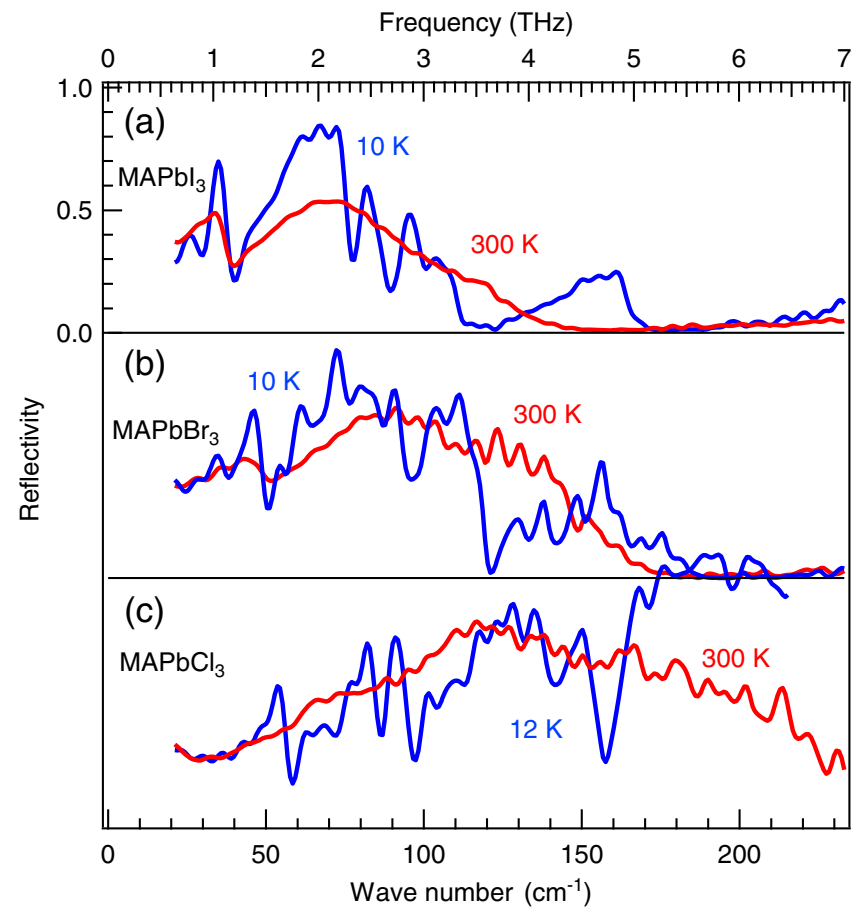

FIG. 1. The reflection coefficients of the $\operatorname{MAPb} X_{3}[X=\mathrm{I}(\mathrm{a})$, $\mathrm{Br}(\mathrm{b})$, and $\mathrm{Cl}$ (c)] single crystals at low temperature (blue curve) and room temperature (red curve). The $\mathrm{THz}$ pulses were $p$ polarized and had an incidence angle of $\theta=30^{\circ}$. single crystals at low temperature and at $300 \mathrm{~K}$ in Figs. 1(b) and 1(c), respectively. By comparing their data with that of $\mathrm{MAPbI}_{3}$, we find that the two reflection peaks at room temperature are shifted to higher frequencies, but at low temperatures the oscillation in the reflection spectra appear at similar wave numbers.

We carefully evaluated the dielectric constant $\epsilon$ from the complex reflection coefficient with a phase compensation [45]. Details are described in the Supplemental Material [37]. The imaginary part $\epsilon_{2}$ of the complex dielectric constant $\epsilon=\epsilon_{1}+i \epsilon_{2}$ for the three different $\mathrm{MAPb} X_{3}$ single crystal samples are shown in Fig. 2 for 10 and $300 \mathrm{~K}$. We summarize the spectra of $\epsilon$ at different temperatures in Figs. S3(a), S4(a), and S5(a) of the Supplemental Material [37]. The dielectric components can be categorized into the following two types [46-52]. The first type exhibits two strong peaks at low wave numbers $\left(<100 \mathrm{~cm}^{-1}\right)$ at room temperature. Their resonance wave numbers depend on the halogen $(X): 30$ and $57 \mathrm{~cm}^{-1}$ for $X=\mathrm{I}, 46$ and $73 \mathrm{~cm}^{-1}$ for $X=\mathrm{Br}$, and 72 and $98 \mathrm{~cm}^{-1}$ for $X=\mathrm{Cl}$. At low temperatures, these two modes split into four modes $[14,17]$. The second type includes the weak peaks that appear in the low-temperature orthorhombic phase in the higher wave number region from 80 to $160 \mathrm{~cm}^{-1}$.

First, we discuss the type of the dielectric components in the lower wave number region. La-o-vorakiat et al. reported two phonon modes related to the lead halide cage at 32 and $63 \mathrm{~cm}^{-1}$ for the room-temperature $\mathrm{MAPbI}_{3}$ thin film [14],

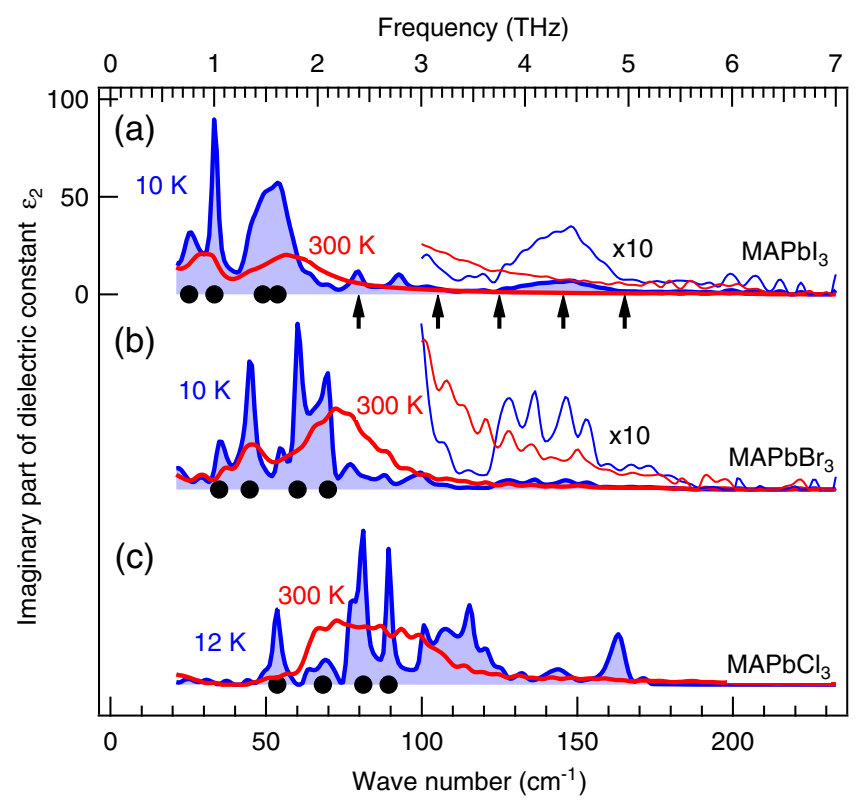

FIG. 2. The imaginary parts of the dielectric constants for the three $\mathrm{MAPb} X_{3}$ single crystals. The black dots indicate the mode positions related to the lead halide cage at low temperature. The black arrows show the positions of the theoretically predicted MA cation modes, reported in Ref. [53]. 
which corresponds well with the peaks observed in Fig. 2(a). The mode at $32 \mathrm{~cm}^{-1}$ is related to the $\mathrm{Pb}-\mathrm{I}-\mathrm{Pb}$ rocking vibration, and the mode at $63 \mathrm{~cm}^{-1}$ is attributed to the $\mathrm{Pb}$-I stretching vibrations $[47,54]$. These two phonon modes shift to higher frequencies upon exchange of I with the lighter halogens $\mathrm{Br}$ and $\mathrm{Cl}$ [15]. Figure 2 also shows that the two high-temperature phonon modes related to the lead halide cages split into the four modes at low temperature. The peak positions of the four modes at low temperature are indicated by the black circles in Fig. 2. The mode splitting below the critical temperature of the tetragonal-to-orthorhombic phase transition has also been predicted by numerical simulations [55] and confirmed with $\mathrm{THz}$ time-domain spectroscopy [14] for $\mathrm{MAPbI}_{3}$. Our results prove that the same mode splitting occurs for all three $\mathrm{MAPb} X_{3}$ crystals.

In the following, we discuss the origin of the dielectric components that were observed at low temperature in the higher wave number range between 80 and $160 \mathrm{~cm}^{-1}$. Based on the results of Raman spectroscopy [47,49], we consider that these peaks are governed by the motion of MA cations. A numerical simulation for $\mathrm{MAPbI}_{3}$ based on a combination of first principles and classical molecular dynamics revealed that some rotational modes lie at 80 , 125 , and $165 \mathrm{~cm}^{-1}$ and the translational mode lies at $105 \mathrm{~cm}^{-1}$ [53]. The spinning of the MA cations around their $\mathrm{CN}$ axis is expected at a wave number of $145 \mathrm{~cm}^{-1}$, but this is an infrared-inactive mode. Our experimental results for $\mathrm{MAPbI}_{3}$ in Fig. 2(a) show a broad peak around $150 \mathrm{~cm}^{-1}$ and two sharp peaks at 100 and $125 \mathrm{~cm}^{-1}$. We assign them to the modes related to MA cations because the weak peaks of the $\mathrm{Br}$ - and $\mathrm{Cl}$-based perovskite single crystals appeared at similar wave numbers: $78,87,100$, $125 \mathrm{~cm}^{-1}$, and from $135 \mathrm{~cm}^{-1}$ to $155 \mathrm{~cm}^{-1}$ in $\mathrm{MAPbBr}_{3}$ [Fig. 2(b)], and 100, 108, 117, 140, and $162 \mathrm{~cm}^{-1}$ in $\mathrm{MAPbCl}_{3}$ [Fig. 2(c)]. Figure 3 shows the two-dimensional image plots of $\epsilon_{2}$ in the $\mathrm{MAPb}_{3}$ single crystals as a function of the temperature (horizontal axis) and wave number (vertical axis). They clarify that the MA cation modes appear at similar wave numbers under low temperatures, in spite of the different halogen. This behavior has also been confirmed with Raman spectra [49].

The absorption peaks above $70 \mathrm{~cm}^{-1}$ are sharp when the MA cations are strongly constrained by the lead halide cage lattice in the low-temperature orthorhombic phase. Theoretical investigations predicted that the mixed organicinorganic modes for frequencies within $70-120 \mathrm{~cm}^{-1}$ are strongly modulated in the high-temperature phase by fast reorientations of the MA cations, while the rotational modes of the MA cation above $120 \mathrm{~cm}^{-1}$ survive [53]. Our experimental results in Fig. 3 show that all weak peaks disappeared in the high-temperature phase or became broader and extended towards the low-frequency region. This result indicates that the observed MA cation modes are very sensitive to the dynamics of the cation reorientation.

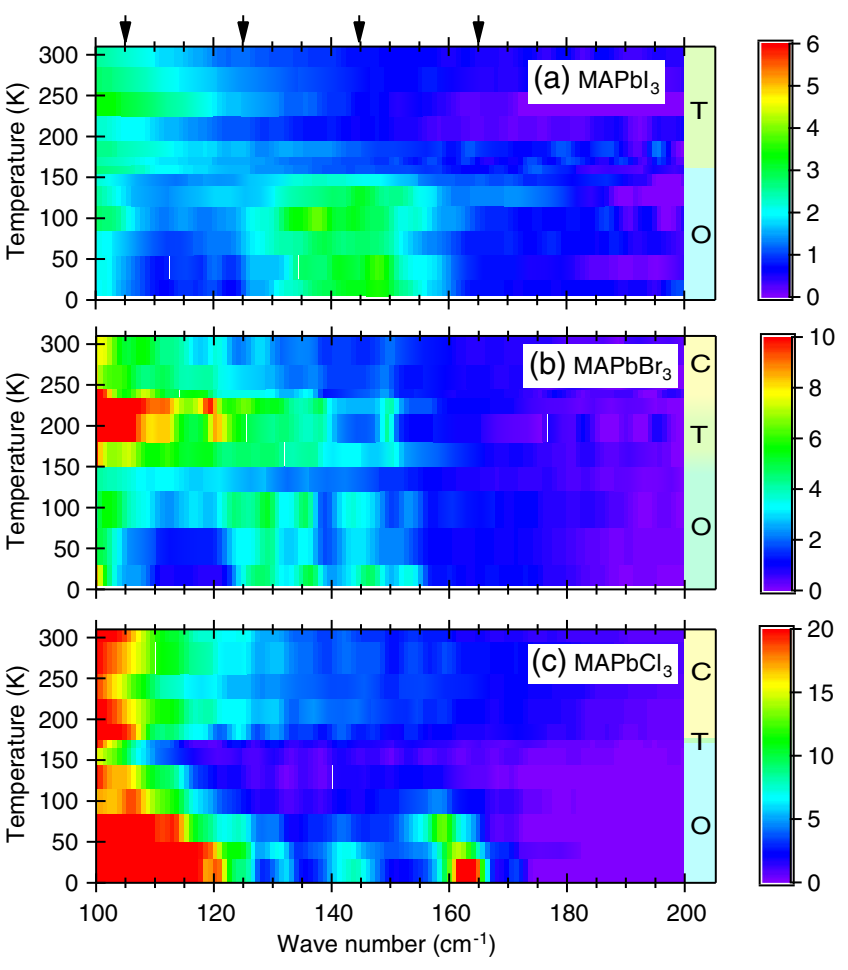

FIG. 3. The 2D image plots of $\varepsilon_{2}$ for $\mathrm{MAPb}_{3}[X=\mathrm{I}$ (a), Br (b), and $\mathrm{Cl}$ (c)] single crystals as a function of the temperature (horizontal axis) and wave number (vertical axis). The labels $\mathrm{O}$, $\mathrm{T}$, and $\mathrm{C}$ represent orthorhombic, tetragonal, and cubic phases, respectively. The black arrows on the top axis show the positions of the theoretically predicted MA cation modes reported in Ref. [53].

Figure 1 showed that the reflection spectra experienced a drastic change under low temperature, which implies a modulation of the Reststrahlen band. Consequently, this also implies the modulation of the longitudinal modes such as the LO phonons. In our experiments, we can easily obtain the energy loss function $\operatorname{Im}(-1 / \epsilon)$, which directly represents the longitudinal excitation [35], from $\epsilon$. The results for the I-, Br-, and Cl-based perovskites are shown in the left-hand panel of Fig. 4. For direct comparison with $\epsilon_{2}$, the spectra of $\operatorname{Im}(-1 / \epsilon)$ are also shown in Figs. S3(b), S4(b), and S5(b) in the Supplemental Material [37]. The peaks of $\operatorname{Im}(-1 / \epsilon)$ at $300 \mathrm{~K}$ lie at $130 \mathrm{~cm}^{-1}$ for $\mathrm{MAPbI}_{3}$ and at $160 \mathrm{~cm}^{-1}$ for $\mathrm{MAPbBr}_{3}$. These wave numbers are almost the same as those reported for the LO phonons [16]. We note that the wave numbers of $\mathrm{LO}$ phonons in $\mathrm{MAPbI}_{3}$ and $\mathrm{MAPbBr}_{3}$ are close to those of the MA cation modes. We can also confirm that each peak of $\operatorname{Im}(-1 / \epsilon)$ is split into two peaks at low temperature: 110 and $165 \mathrm{~cm}^{-1}$ for $\mathrm{MAPbI}_{3}$ and, similarly, 120 and $180 \mathrm{~cm}^{-1}$ for $\mathrm{MAPbBr}_{3}$. The closed circles in Figs. 4(d) and 4(e) show the peak wave numbers of $\operatorname{Im}(-1 / \epsilon)$ as a function of the temperature for and $\mathrm{MAPbBr}_{3}$, respectively. $\mathrm{MAPbCl}_{3}$ is not analyzed here because we were not able to confirm a peak for $\mathrm{MAPbCl}_{3}$ within our detection range. In principle, the 

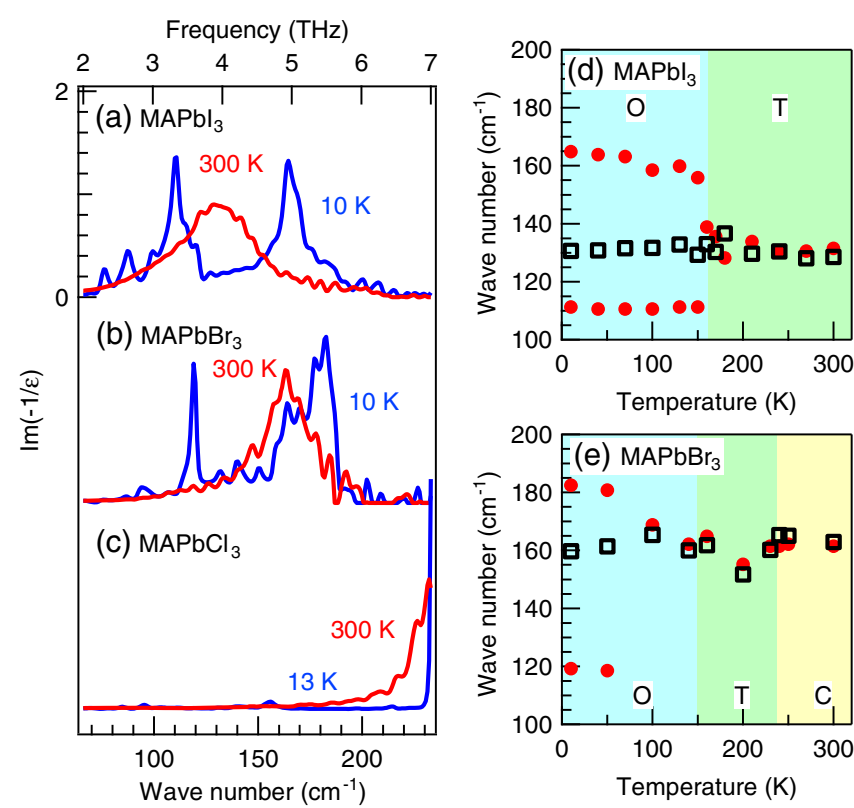

FIG. 4. The left-hand panel shows the energy loss function $\operatorname{Im}(-1 / \epsilon)$ of the $\mathrm{MAPb}_{3}[X=\mathrm{I}(\mathrm{a}), \mathrm{Br}(\mathrm{b})$, and $\mathrm{Cl}$ (c) ] single crystals at different temperatures. The closed circles in the righthand panels show the peak wave numbers in (d) $\mathrm{MAPbI}_{3}$ and (e) $\mathrm{MAPbBr}_{3}$ single crystals as a function of the temperature. The open squares show the wave numbers of effective LO phonon. The labels $\mathrm{O}$, T, and $\mathrm{C}$ represent orthorhombic, tetragonal, and cubic phases, respectively.

mode splitting observed at low temperatures can be attributed to either a lower symmetry of the orthorhombic perovskite structure or a coupling with the MA cation. We consider that the latter effect is responsible for the LO phonon splitting because the splitting of the transversal optical (TO) phonons related to the $\mathrm{Pb} X_{6}$ cage modes appears below $100 \mathrm{~cm}^{-1}$ at low temperatures $[14,17,47,49,53]$. In case of a LO phonon splitting induced by low symmetry, this would be accompanied by a LO phonon that appears between the two TO phonon resonances in addition to the $\mathrm{LO}$ phonon resonances at frequencies above $100 \mathrm{~cm}^{-1}$. However, such a phonon is not observed in the experiment. In addition, we compared the spectra of $\operatorname{Im}(-1 / \varepsilon)$ of a $\mathrm{CsPBr}_{3}$ pellet with that of a $\mathrm{MAPbBr}_{3}$ crystal [37], and confirmed the contribution of the $A$-site organic cation to the LO phonon.

We analyzed the multiple LO phonon modes by replacing the interaction with that of a single effective LO phonon [56]. The details of our analysis of the $-1 / \epsilon$ spectra are described in the Supplemental Material [37]. The evaluated wave numbers of the effective LO phonons are shown in Figs. 4(d) and 4(e) with the open squares. They prove that the effective LO phonon wave number is almost constant $\left(131 \mathrm{~cm}^{-1}\right.$ for $\mathrm{MAPbI}_{3}$ and $162 \mathrm{~cm}^{-1}$ for $\left.\mathrm{MAPbBr}_{3}\right)$ despite the spectral distortion of $-1 / \epsilon$ at low temperatures. We also show the derived coupling parameters $W_{e}$ in Fig. S8 (b) of the Supplemental Material [37], which indicate the absence of a temperature dependence. These results demonstrate that the influence of the MA cations to the Fröhlich interaction remains even at high temperatures when the MA cations are rotating.

One hypothesis for the interesting optoelectronic properties of the perovskite materials is the formation of a large polaron [9]. The collective motion of the MA cations has been proposed to screen the electron-hole Coulomb potential, providing protection against various interactions that lead to nonradiative recombination. Our experimental results show that the spectrum of the MA cation's motion at high temperatures is so broad that it increases the damping of the LO phonon mode via mode coupling. Therefore, the large polaronic effects can be suppressed.

Recent studies on luminescence from $\mathrm{MAPbI}_{3}$ and $\mathrm{MAPbBr}_{3}$ suggest that the spectral broadening of the exciton is governed by the Fröhlich interaction rather than by charged impurity scattering [11,12]. Since only an accurate LO phonon wave number allows us to interpret the physics correctly, we have to verify our values with an additional PL experiment. Figures 5(a) and 5(b) show the $\mathrm{PL}$ spectra from the $\mathrm{MAPbI}_{3}$ and $\mathrm{MAPbBr}_{3}$ single crystals. We provide no data for $\mathrm{MAPbCl}_{3}$ because we cannot compare the temperature dependence of the PL spectrum with that of the $\operatorname{Im}(-1 / \epsilon)$ spectrum. The detailed temperature dependence of the PL is provided in Fig. S9 of the Supplemental Material [37]. The PL peak positions
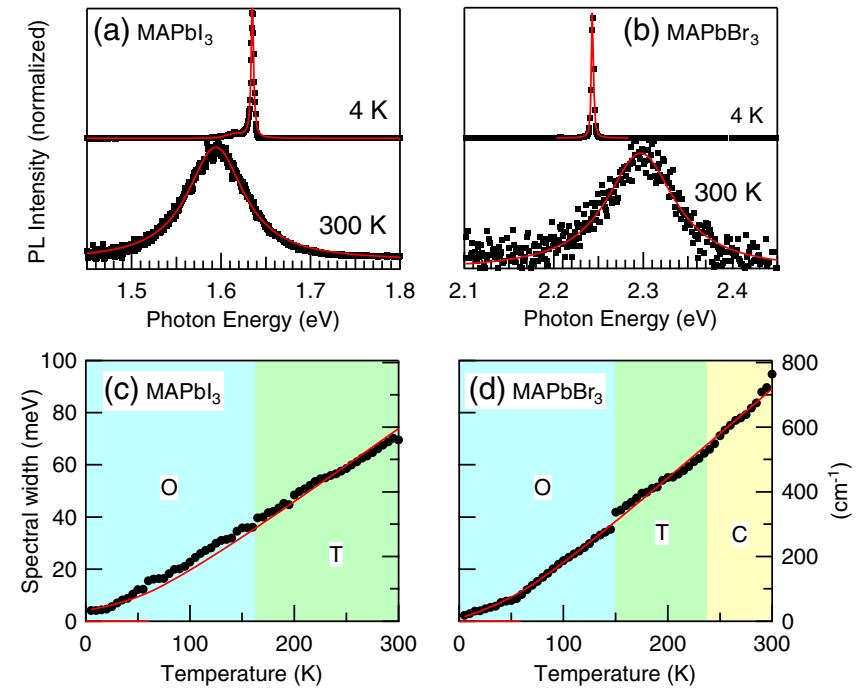

FIG. 5. (a) The PL spectra from the $\mathrm{MAPbI}_{3}$ and (b) $\mathrm{MAPbBr}$ single crystals at different temperatures. (c),(d) The corresponding spectral widths of the luminescence from the free exciton as a function of the temperature. The red curves show the predicted bandwidth including acoustic and LO phonon interaction. The parameters $\Gamma_{0} / h c, \gamma_{\mathrm{LO}} / h c, E_{\mathrm{LO}} / h c$, and $\gamma_{\mathrm{ac}} / h c$ are $32 \mathrm{~cm}^{-1}$, $310 \mathrm{~cm}^{-1}, 131 \mathrm{~cm}^{-1}$, and $0.7 \mathrm{~cm}^{-1} / \mathrm{K}$ for $X=\mathrm{I}$, and $16 \mathrm{~cm}^{-1}$, $505 \mathrm{~cm}^{-1}, 162 \mathrm{~cm}^{-1}$, and $1.0 \mathrm{~cm}^{-1} / \mathrm{K}$ for $X=\mathrm{Br}$. The labels $\mathrm{O}$, $\mathrm{T}$, and $\mathrm{C}$ represent the orthorhombic, tetragonal, and cubic phases, respectively. 
shift with temperature due to the temperature dependence of the band-gap energy [24]. Here, we evaluated the bandwidth of the free exciton peak in the PL spectrum using one Lorentzian function at the highenergy side. The full width at half maximum of the Lorentzian component is given in Figs. 5(c) and 5(d) for $\mathrm{MAPbI}_{3}$ and $\mathrm{MAPbBr}_{3}$, respectively. The spectral width of the free exciton can be expressed as $\Gamma(T)=$ $\Gamma_{0}+\gamma_{\mathrm{LO}} /\left[\exp \left(-E_{\mathrm{LO}} / k_{B} T\right)-1\right]+\gamma_{\mathrm{ac}} T$, with the temperature-independent spectral width $\Gamma_{0}$, the coupling strength for the LO phonons $\left(\gamma_{\mathrm{LO}}\right)$, and that for the acoustic phonons $\left(\gamma_{\mathrm{ac}}\right)$. Based on this model, the spectral bandwidths at $T=0 \mathrm{~K}$ are $\Gamma_{0}^{\mathrm{I}}=32 \mathrm{~cm}^{-1}$ and $\Gamma_{0}^{\mathrm{Br}}=16 \mathrm{~cm}^{-1}$. These widths are governed by the acoustic phonon interactions $\left(\gamma_{\mathrm{ac}}^{\mathrm{I}} / h c=0.7 \mathrm{~cm}^{-1} / \mathrm{K}[12]\right.$ and $\left.\gamma_{\mathrm{ac}}^{\mathrm{Br}} / h c=1.0 \mathrm{~cm}^{-1} / \mathrm{K}\right)$ below the threshold temperature $T=E_{\mathrm{LO}} / k_{B}$, and by the Fröhlich interaction above this temperature. The fitting results are shown with the red curves in Figs. 5(c) and 5(d) for $\mathrm{MAPbI}_{3}$ and $\mathrm{MAPbBr}_{3}$, respectively. We obtained $\gamma_{\mathrm{LO}}^{\mathrm{I}} / h c=310 \mathrm{~cm}^{-1}$ for $\mathrm{MAPbI}_{3}$ and $\gamma_{\mathrm{LO}}^{\mathrm{Br}} / h c=505 \mathrm{~cm}^{-1}$ for $\mathrm{MAPbBr}_{3}$. These results prove the validity of the $\mathrm{LO}$ phonon wave numbers evaluated in Fig. 4.

It is known that the LO phonon also influences the carrier mobility [57]. The temperature dependence of the mobility of photoexcited carriers has been investigated in $\mathrm{MAPbI}_{3}$ $[31,58]$, and there are no reports on a discontinuity of the mobility at the phase transition temperature. These results also support our evaluated temperature dependence of the effective LO phonon wave number.

In conclusion, we evaluated the $\operatorname{Im}(-1 / \epsilon)$ spectra with the $\mathrm{THz}$ time-domain reflection spectroscopy for three $\mathrm{MAPb}_{3}(X=\mathrm{I}, \mathrm{Br}$, and $\mathrm{Cl})$ single crystals and found that the coupling between the MA cation modes and LO phonon mode derived from lead halide cages induces a mode splitting at low temperatures and a damping of the LO phonon mode at high temperatures. It indicates that the additional freedom of the $A$-site cation motion in the perovskite crystal modulates the spectrum of the LO phonon. The temperature dependence of $\epsilon$ indicates that the modes of the MA cations are very sensitive to dynamic reorientation in the high-temperature phase. Such a change should influence phenomena that are sensitive to the phonon distribution, e.g., the phonon bottleneck [10]. However, the wave number of the effective single LO phonon, $131 \mathrm{~cm}^{-1}$ for $\mathrm{MAPbI}_{3}$ and $162 \mathrm{~cm}^{-1}$ for $\mathrm{MAPbBr}_{3}$, is independent of the temperature, which is supported by the temperature-dependent PL spectra. These results aid the understanding of the intrinsic influences of the MA cations' motions on the carrier-phonon interaction in $\mathrm{MAPbX}_{3}$ under photoexcitation and have important implications for perovskite semiconductors.

This work was financially supported by JST CREST Grant No. JPMJCR16N3 and JSPS KAKENHI Grant No. $15 \mathrm{H} 03579$. *mnagai@mp.es.osaka-u.ac.jp

†yasuyamada@chiba-u.jp

kanemitu@scl.kyoto-u.ac.jp

[1] World-record solar-cell efficiencies, https://www.nrel.gov/ pv/assets/images/efficiency-chart.png.

[2] S. D. Stranks, G. E. Eperon, G. Grancini, C. Menelaou, M. J. P. Alcocer, T. Leijtens, L. M. Herz, A. Petrozza, and H. J. Snaith, Science 342, 341 (2013).

[3] Q. Lin, A. Armin, R. C. R. Nagiri, P. L. Burn, and P. Meredith, Nat. Photonics 9, 106 (2015).

[4] Z.-K. Tan, R. S. Moghaddam, M. L. Lai, P. Docampo, R. Higler, F. Deschler, M. Price, A. Sadhanala, L. M. Pazos, D. Credgington, F. Hanusch, T. Bein, H. J. Snaith, and R. H. Friend, Nat. Nanotechnol. 9, 687 (2014).

[5] H. Zhu, Y. Fu, F. Meng, X. Wu, Z. Gong, Q. Ding, M. V. Gustafsson, M. T. Trinh, S. Jin, and X.-Y. Zhu, Nat. Mater. 14, 636 (2015).

[6] F. Li, C. Ma, H. Wang, W. Hu, W. Yu, A. D. Sheikh, and T. Wu, Nat. Commun. 6, 8238 (2015).

[7] P. Y. Yu and M. Cardona, Fundamentals of Semiconductors (Springer, Berlin, 1996).

[8] T. M. Brenner, D. A. Egger, A. M. Rappe, L. Kronik, G. Hodes, and D. Cahen, J. Phys. Chem. Lett. 6, 4754 (2015).

[9] X.-Y. Zhu and V. Podzorov, J. Phys. Chem. Lett. 6, 4758 (2015).

[10] Y. Yang, D. P. Ostrowski, R. M. France, K. Zhu, J. van de Lagemaat, J. M. Luther, and M. C. Beard, Nat. Photonics 10, 53 (2016).

[11] A. D. Wright, C. Verdi, R. L. Milot, G. E. Eperon, M. A. Pérez-Osorio, H. J. Snaith, F. Giustino, M. B. Johnston, and L. M. Herz, Nat. Commun. 7, 11755 (2016).

[12] L. Q. Phuong, Y. Nakaike, A. Wakamiya, and Y. Kanemitsu, J. Phys. Chem. Lett. 7, 4905 (2016).

[13] J. Tilchin, D. N. Dirin, G. I. Maikov, A. Sashchiuk, M. V. Kovalenko, and E. Lifshitz,ACS Nano 10, 6363 (2016).

[14] C. La-o-vorakiat, H. Xia, J. Kadro, T. Salim, D. Zhao, T. Ahmed, Y. M. Lam, J.-X. Zhu, R. A. Marcus, M.-E. MichelBeyerle, and E. E. M. Chia, J. Phys. Chem. Lett. 7, 1 (2016).

[15] R. G. Niemann, A. G. Kontos, D. Palles, E. I. Kamitsos, A. Kaltzoglou, F. Brivio, P. Falaras, and P. J. Cameron, J. Phys. Chem. C 120, 2509 (2016).

[16] M. Sendner, P. K. Nayak, D. A. Egger, S. Beck, C. Müller, B. Epding, W. Kowalsky, L. Kronik, H. J. Snaith, A. Pucci, and R. Lovrinčić, Mater. Horiz. 3, 613 (2016).

[17] D. Zhao, J. M. Skelton, H. Hu, C. La-o-vorakiat, J.-X. Zhu, R. A. Marcus, M.-E. Michel-Beyerle, Y. M. Lam, A. Walsh, and E. E. M. Chia, Appl. Phys. Lett. 111, 201903 (2017).

[18] M. R. Filip, G. E. Eperon, H. J. Snaith, and F. Giustino, Nat. Commun. 5, 5757 (2014).

[19] V. D'Innocenzo, A. R. S. Kandada, M. De Bastiani, M. Gandini, and A. Petrozza, J. Am. Chem. Soc. 136, 17730 (2014).

[20] O. G. Reid, M. Yang, N. Kopidakis, K. Zhu, and G. Rumbles, ACS Energy Lett. 1, 561 (2016).

[21] Y. Kanemitsu, J. Mater. Chem. C 5, 3427 (2017).

[22] Y. Yamada, T. Yamada, and Y. Kanemitsu, Bull. Chem. Soc. Jpn. 90, 1129 (2017).

[23] C. Wehrenfennig, M. Liu, H. J. Snaith, M. B. Johnston, and L. M. Herz, APL Mater. 2, 081513 (2014). 
[24] Y. Yamada, T. Nakamura, M. Endo, A. Wakamiya, and Y. Kanemitsu, IEEE J. Photovoltaics 5, 401 (2015).

[25] R. L. Milot, G. E. Eperon, H. J. Snaith, M. B. Johnston, and L. M. Herz, Adv. Funct. Mater. 25, 6218 (2015).

[26] H. Tahara, M. Endo, A. Wakamiya, and Y. Kanemitsu, J. Phys. Chem. C 120, 5347 (2016).

[27] L. Q. Phuong, Y. Yamada, M. Nagai, N. Maruyama, A. Wakamiya, and Y. Kanemitsu, J. Phys. Chem. Lett. 7, 2316 (2016).

[28] N. Onoda-Yamamuro, T. Matsuo, and H. Suga, J. Phys. Chem. Solids 53, 935 (1992).

[29] Y. Yamada, T. Nakamura, M. Endo, A. Wakamiya, and Y. Kanemitsu, Appl. Phys. Express 7, 032302 (2014).

[30] M. I. Dar, G. Jacopin, S. Meloni, A. Mattoni, N. Arora, A. Boziki, S. M. Zakeeruddin, U. Rothlisberger, and M. Grätzel, Sci. Adv. 2, e1601156 (2016).

[31] O. Knop, R. E. Wasylishen, M. A. White, T. S. Cameron, and M. J. M. V. Oort, Can. J. Chem. 68, 412 (1990).

[32] A. A. Bakulin, O. Selig, H. J. Bakker, Y. L. A. Rezus, C. Mueller, T. Glaser, R. Lovrincic, Z. Sun, Z. Chen, A. Walsh, J. M. Frost, and T. L. C. Jansen, J. Phys. Chem. Lett. 6, 3663 (2015).

[33] A. M. A. Leguy, J. M. Frost, A. P. McMahon, V. G. Sakai, W. Kochelmann, C. Law, X. Li, F. Foglia, A. Walsh, B. C. O’Regan, J. Nelson, J. T. Cabral, and P. R. F. Barnes, Nat. Commun. 6, 7124 (2015).

[34] P. U. Jepsen and B. M. Fischer, Opt. Lett. 30, 29 (2005).

[35] F. Gervais and B. Piriou, Phys. Rev. B 10, 1642 (1974).

[36] M. Saliba, T. Matsui, J.-Y. Seo, K. Domanski, J.-P. CorreaBaena, M. K. Nazeeruddin, S. M. Zakeeruddin, W. Tress, A. Abate, A. Hagfeldt, and M. Grätzela, Energy Environ. Sci. 9, 1989 (2016).

[37] See Supplemental Material at http://link.aps.org/ supplemental/10.1103/PhysRevLett.121.145506, which includes Refs. [38-43], for details of the sample preparation, setup of THz reflection spectroscopy, analysis methods, and PL spectroscopy.

[38] D. Shi, V. Adinolfi, R. Comin, M. Yuan, E. Alarousu, A. Buin, Y. Chen, S. Hoogland, A. Rothenberger, K. Katsiev, Y. Losovyj, X. Zhang, P. A. Dowben, O. F. Mohammed, E. H. Sargent, and O. M. Bakr, Science 347, 519 (2015).

[39] Y. Yamada, M. Hoyano, R. Akashi, K. Oto, and Y. Kanemitsu, J. Phys. Chem. Lett. 8, 5798 (2017).

[40] Y. Yamada, T. Yamada, L. Q. Phuong, N. Maruyama, H. Nishimura, A. Wakamiya, Y. Murata, and Y. Kanemitsu, J. Am. Chem. Soc. 137, 10456 (2015).
[41] T. Yamada, Y. Yamada, Y. Nakaike, A. Wakamiya, and Y. Kanemitsu, Phys. Rev. Applied 7, 014001 (2017).

[42] G. Yamashita, E. Matsubara, M. Nagai, C. Kim, H. Akiyama, Y. Kanemitsu, and M. Ashida, Appl. Phys. Lett. 110, 071108 (2017).

[43] G. Hecht, Optics, 5th ed. (Pearson, Boston, 2016).

[44] D. A. Valverde-Chávez, C. S. Ponseca, Jr., C. C. Stoumpos, A. Yartsev, M. G. Kanatzidis, V. Sundströmb, and D. G. Cooke, Energy Environ. Sci. 8, 3700 (2015).

[45] S. Nashima, O. Morikawa, K. Takata, and M. Hangyo, Appl. Phys. Lett. 79, 3923 (2001).

[46] C. Quarti, G. Grancini, E. Mosconi, P. Bruno, J. M. Ball, M. M. Lee, H. J. Snaith, A. Petrozza, and F. D. Angelis, J. Phys. Chem. Lett. 5, 279 (2014).

[47] F. Brivio, J. M. Frost, J. M. Skelton, A. J. Jackson, O. J. Weber, M. T. Weller, A. R. Goñi, A. M. A. Leguy, P. R. F. Barnes, and A. Walsh, Phys. Rev. B 92, 144308 (2015).

[48] M. Ledinský, P. Löper, B. Niesen, J. Holovský, S.-J. Moon, J.-H. Yum, S. D. Wolf, A. Fejfar, and C. Ballif, J. Phys. Chem. Lett. 6, 401 (2015).

[49] A. M. A. Leguy, A. R. Goñi, J. M. Frost, J. Skelton, F. Brivio, X. Rodríguez-Martínez, O. J. Weber, A. Pallipurath, M. I. Alonso, M. Campoy-Quiles, M. T. Weller, J. Nelson, A. Walsh, and P. R. F. Barnes, Phys. Chem. Chem. Phys. 18, 27051 (2016).

[50] T. Ivanovska, C. Quarti, G. Grancini, A. Petrozza, F. D. Angelis, A. Milani, and G. Ruani, Chem. Sus. Chem. 9, 2994 (2016).

[51] Y. Guo, O. Yaffe, D. W. Paley, A. N. Beecher, T. D. Hull, G. Szpak, J. S. Owen, L. E. Brus, and M. A. Pimenta, Phys. Rev. Matter 1, 042401(R) (2017).

[52] E. Mosconi, C. Quarti, T. Ivanovska, G. Ruani, and F. De Angelis, Phys. Chem. Chem. Phys. 16, 16137 (2014).

[53] A. Mattoni, A. Filippetti, M. I. Saba, C. Caddeo, and P. Delugas, J. Phys. Chem. Lett. 7, 529 (2016).

[54] C. Wehrenfennig, M. Liu, H. J. Snaith, M. B. Johnston, and L. M. Herz, Energy Environ. Sci. 7, 2269 (2014).

[55] T. Ahmed, C. La-o-vorakiat, T. Salim, Y. M. Lam, E. E. M. Chia, and J.-X. Zhu, Europhys. Lett. 108, 67015 (2014).

[56] R. W. Hellwarth and I. Biaggio, Phys. Rev. B 60, 299 (1999).

[57] L. M. Herz, ACS Energy Lett. 2, 1539 (2017).

[58] M. Karakus, S. A. Jensen, F. D’Angelo, D. Turchinovich, M. Bonn, and E. Cánovas, J. Phys. Chem. Lett. 6, 4991 (2015). 